\title{
Téoros
}

Revue de recherche en tourisme

\section{AITQ, formation et reconnaissance professionnelle}

\section{Roger Nadeau}

Volume 11, numéro 1, mars 1992

La formation en tourisme : à la croisée des chemins

URI : https://id.erudit.org/iderudit/1078950ar

DOI : https://doi.org/10.7202/1078950ar

Aller au sommaire du numéro

Éditeur(s)

Université du Québec à Montréal

ISSN

0712-8657 (imprimé)

1923-2705 (numérique)

Découvrir la revue

Citer cet article

Nadeau, R. (1992). AITQ, formation et reconnaissance professionnelle. Téoros, 11(1), 42-43. https://doi.org/10.7202/1078950ar d'utilisation que vous pouvez consulter en ligne.

https://apropos.erudit.org/fr/usagers/politique-dutilisation/ 


\section{AITQ, formation et}

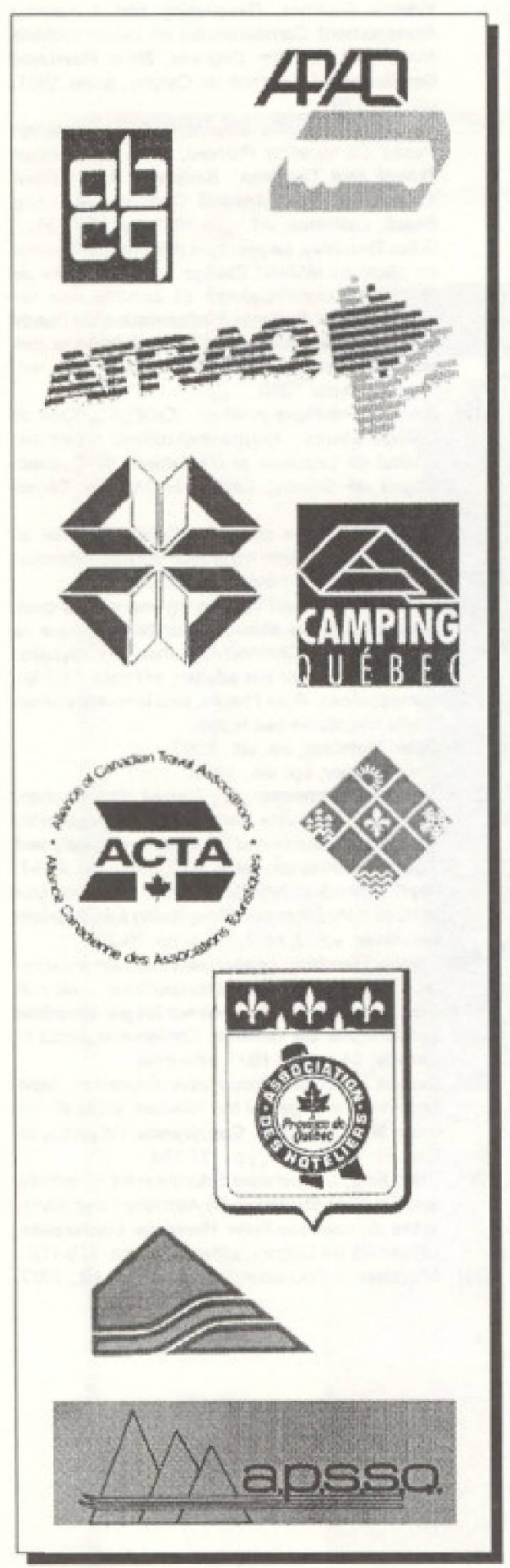

Monsieur Roger Nadeau est directeur du département de geographie et teldddection a r'Universite de Sherbrooke.
Depuis déjà quelques décennies, les diagnostics que l'on porte sur l'état de santé de l'industrie touristique du Québec font ressortir des lacunes évidentes dans le domaine de la concertation. Les gens du tourisme sont des individualistes, prétend-on. Ils ont bien des difficultés à travailler ensemble, ajoute-t-on. L'industrie touristique du Québec, c'est 20 000 entreprises et 75000 emplois équivalents plein temps. Ces deux dernières données expliquent sans doute dans une bonne mesure les attitudes signalées précédemment. Un très grand nombre d'entreprises dans le domaine du tourisme sont très petites, comptant souventun, deux ou trois employés seulement; elles fonctionnent sur une base artisanale ou familiale. Chacun trime dur pour que sa boîte fonctionne bien et le voisin est un concurrent plutồt qu'un collègue qui oeuvre dans le même domaine.

Cet isolement relatif ne permet pas aux intervenants d'avoir une vue d'ensemble sur l'état de l'industrie dans leur région et encore moins au Québec. Ils seront donc souventen retard sur les ajustements à faire en fonction des nouvelles tendances du marche. Tout le monde s'entend la-dessus: il y a un besoin criant de formation continue chez les intervenants touristiques, non seulement auprès de la préposée à l'accueil ou aux cuisines mais aussi auprès des gestionnaires ou des patrons des entreprises.

Évidemment, l'avènement des ATR, il y a une douzaine d'années, a quelque peu amélioré cette situation, en regroupant, en consultant, en informant et en permettant aux intervenants de se connaitre etde se parler, au moins sur une base régionale. Voila qui est un progrès important mais très inégal selon la personnalité des ATR. En Estrie, par exemple, sur 300 membres, 45 seulement se sont présentés à l'assemblée générale de leur ATR en 1991, signe très net d'un malaise profond et d'un sentiment d'appartenance qui se dégrade rapidement. Il y a des structures qui sont excellentes mais qui sont détournées de leur vraie mission par le facteur humain...
Bien sûr, ces cas ne doivent pas être généralisés car il faut bien reconnaître que dans l'ensemble, les ATR ont assez de respect pour les intervenants touristiques pour leur transmetire l'information et les services qui leurpermettront d'être plus performants, non seulement en terme de marketing mais aussi en terme de développement, de gestion (financière ou de personnel), de qualité totale, d'innovation ou de formation. Sur ce dernier volet, il est clair que la structure des ATR offredespossibilitésd'intervention de grande qualité, pour autant qu'on s'en donne la peine - et donc qu'on y croit aux vertus de la formation continue et du perfectionnement.

Le modele classique de formation initié par les ATR est sans doute celui qui s'adresse annuellement aux agents d'information, préposés à l'accueil et guides touristiques oeuvrant dans le cadre des bureaux d'information touristiques (BIT). Cette formation est non seulement essentielle pour assurer le professionnalisme de la fonction mais aussi parce qu'elle est porteuse d'une forme d'accréditation ou dereconnaissance formelle du travailleuroude latravailleuseen tourisme. Voilà une facette de l'activité de formation quis' averefort importante dans un monde où l'intervenant touristique, dans la plupart des cas, ne jouit d'aucune reconnaissance professionnelle formelle. Onmetdoncenrelation ici trois composantes associées a la problématique en cours: un groupement/ Association, une bonne formation et la reconnaissance professionnelle valorisant l'intervenant en tourisme. Reconnaissant cetterelation, leministère du Tourisme verse, par protocole, al l'ensemble des Associations touristiques régionales du Québec, un budget annuel exclusivement réservé au personnel des ATR. L'initiation par leMinistère de ce processus exemplaire indique l' intérêt qu'il porte à la question et devrait être un incitatif pour les ATR a aller plus loin, vers l'Excellence, ... clame-t-on souvent, comme s'il ne s'agissait que d'un voeu pieux.

Jadis, le Centre d'études du tourisme (CET) etl'Association techniquedu tourisme(ATT), souvent associés au ministère du Tourisme, initièrent les premières activités structurées de formation; aujourd' hui, cesont Téoros, les 
ATR et l'ATRAQ, ou les grandes associations sectorielles, notamment. Depuis peu, certaines ATR ont même pris l'initiative d'organiser des colloques nationaux avecune thematique assez pointue. Par exemple, il y a quelques années, l'ATR de l'Estrie organisait un colloque sur les manifestations evénementielles a caractère touristique; à la fin d'avril 1992, l'ATR ChaudièreAppalaches organise le 1er colloque national tourisme et circuit, tandis que l'ATR du Bas St-Laurent, en collaboration avec Pohénégamook Santé Plein Airetl'Institut du Plein Air Québécois, annonce un colloque national sur l'écotourisme en novembre 1992. Voila des initiatives extrêmement intéressantes, qui diffusent une information spécialisée auprès des intervenants. Tout cela parce que l'on devient de plus en plus conscient des lacunes de formation qui affectent une partie importante de la main-d'oeuvre oeuvrant au sein de l'industrie touristique du Québec. Or, il est illusoire de parler d'excellence sans l'associerà un haut niveau de compétence sereine.

L'ensemble des ATR, regroupées au sein de l'ATRAQ, rassemblent plusieurs dizaines demilliers d'intervenants touristiques de tout le Québec. L'ATRAQ est donc un intervenant majeur du fait qu'elle rejoint les 19 régions touristiques du Québec, ce qui ne lui permet pas, pour autant, de pouvoir parler au nom des grandes associations sectorielles qui sont plutôt bien compartimentées et relativement étanches aux influences des voisins. Par exemple, l' Association des restaurateurs se sent fort peu concemée par celle des Agents de voyages ou par celle des Pourvoyeurs en chasse et pêche; de même, l'Association des hôteliers de la province de Québec se donne rarement l'occasion d'echanger avec celle des Terrains de camping, des proprietaires des Compagnies d'autobus, des Transporteurs aériens nationaux ou des stations de ski, etc. Tous ces intervenants majeurs travaillent au sein de la même industrie, celle du tourisme québécois, et pourtant, ils évoluent habituellement en marge les uns des autres, et même en marge de l'ATRAQ. Le résultat, c'est que notre industrie touristique est éparpillee, "écartillee", écartelée! Contrairement au monde rural qui s'exprime en bloc par le président de l'UPA, contrairement au monde industriel qui s'exprime en bloc par le representant du Conseil du patronat, le tourisme, une industrie de 4 milliards de dollars, affaiblit considérablement son message en multipliant ses voix... et ses messages. Il y a dejà fort longtemps que l'on souhaite la mise en place de l'Association de l'industrie tou- ristique du Québec. En 1992, le Québec est encore la seule province canadienne à ne pas disposer d'une voix unifiée pour défendre et parler au nom de toute son industrie touristique. C'est triste... même si des signes encourageants éclairent l'horizon: des gestes concrets de regroupement et de concertation intersectoriels ont été posé récemment, sur une base ponctuelle et circonstancielle. Quand le ministère du Tourisme a indiqué son intention d'imposer une nouvelle taxe sur les chambres d'hôtel, une dizaine d'associations sectorielles ont fait front commun pour contrer ce projet... qui a fait long feu. Et la Coalition de l'industrie touristique québécoise existe depuis un an et regroupe plusieurs associations sectorielles, les ATR et l'ATRAQ; ensemble, et au nom de l'industrie, ces regroupements font front commun sur des dossiers comme la TVQ de notre bien aimé Ministre, sur les casinos et les budgets de promotion. Si on peut apprendre à travailler ensemble, la Coalition pourrait peut-être conduire enfin à une structure de type AITQ, ce qui serait un pas magistral pour la reconnaissance de l'industrie.

Cette réflexion nous ramène finalement à la trilogie signalée précédemment, à savoir: regroupement - formation - reconnaissance professionnelle. La mise sur pied d'organismes regroupant les intervenants touristiques, tels les ATR, l' AITQ - ou la CITQ - est essentielle mais insuffisante à susciter une vraie reconnaissance professionnelle des gens du tourisme. L'AITQ devra d'abord faire la preuve de son propre professionnalisme, de sa rigueur, de sa capacité à bien établir la place de l'industrie touristique au sein de l'économie du Québec, avec bien sûr, les moyens financiers nécessaire pour y arriver. Voilà un pas important vers la valorisation des gens du tourisme. Dans un deuxième temps, l'AITQ devra se préoccuper en priorité de la formation du personnel en fonction des orientations de développement que l'industrie ce sera donnée. Cette structure offre un potentiel fantastique au niveau de la formation, autant pour les gens en milieu de travail (éducation permanente ou formation continue) que pour les étudiants qui se spécialisent en tourisme au Cégep ou à l'Université. L'AITQ, de plus, pourrait avoir un droit de regard sur le contenu des programmes de formation; elle pourrait ainsi garantir la compétence des travailleurs qui obtiendraient alors leur reconnaissance professionnelle. On ne serait pas alors très loin de la Corporation professionnelle des métiers du tourisme.
Mais, bien sûr, je rêve ici, comme un vrai professeur d'université! Est-ce que l' industrie touristique du Québec a suffisamment de maturité pour pouvoir réaliser ces rêves? $f$

\section{NOTES ET RÉFÉRENCES}

(1) Patrick Cluzeau, Lo Qubbec touristique - Indicatours eur los marchbs ot eur los secteurs tourietiquee do 1980 d 1990, Les Publications du Québec, ministère du Tourisme, Québec, 1991, 552 pages.

(2) André Vallerand (ministre), Enoncé de politique on matiere de tourieme, document de réflexion, Gouvernement du Québec, ministère du Tourisme, 1991, 70 pages.

(3) Ministère du Tourisme du Québec, Dee salsone ot des gens: lo partenarlat, force motrice de Vinduetris tourietique. Énoncé de politique ot plan d'action on matiore de tourieme, Gouvernement du Québecc, Québec, 1992، 96 pages.

(4) La Coalition de l'industrie touristique du Québec (CITQ), Pour une relance Immodlate de tourisme québecols, CITQ. Montréal, 1991, 15 pages. 